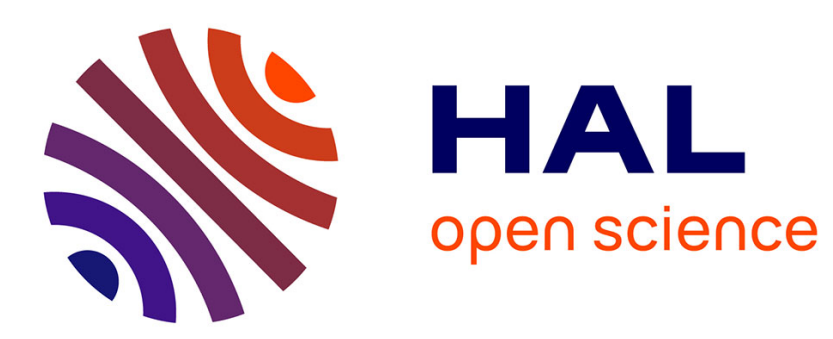

\title{
Sperm-dependent parthenogens delay the spatial expansion of their sexual hosts
}

\author{
K. Janko, J. Eisner
}

\section{To cite this version:}

K. Janko, J. Eisner. Sperm-dependent parthenogens delay the spatial expansion of their sexual hosts. Journal of Theoretical Biology, 2009, 261 (3), pp.431. 10.1016/j.jtbi.2009.08.012 . hal-00559153

\section{HAL Id: hal-00559153 \\ https://hal.science/hal-00559153}

Submitted on 25 Jan 2011

HAL is a multi-disciplinary open access archive for the deposit and dissemination of scientific research documents, whether they are published or not. The documents may come from teaching and research institutions in France or abroad, or from public or private research centers.
L'archive ouverte pluridisciplinaire HAL, est destinée au dépôt et à la diffusion de documents scientifiques de niveau recherche, publiés ou non, émanant des établissements d'enseignement et de recherche français ou étrangers, des laboratoires publics ou privés. 


\section{Author's Accepted Manuscript}

Sperm-dependent parthenogens delay the spatial expansion of their sexual hosts

K. Janko, J. Eisner

PII:

S0022-5193(09)00376-2

DOI: doi:10.1016/j.jtbi.2009.08.012

Reference: YJTBI 5671

To appear in:

Journal of Theoretical Biology

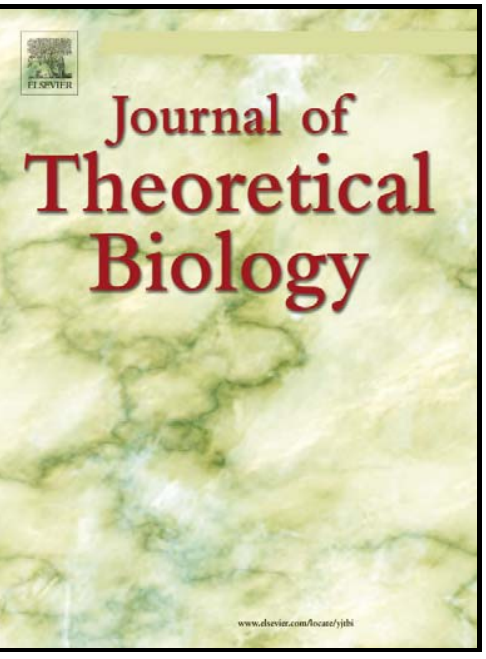

www.elsevier.com/locate/yjtbi

Received date: $\quad 6$ March 2009

Revised date: $\quad 1$ August 2009

Accepted date: 10 August 2009

Cite this article as: K. Janko and J. Eisner, Sperm-dependent parthenogens delay the spatial expansion of their sexual hosts, Journal of Theoretical Biology, doi:10.1016/j.jtbi.2009.08.012

This is a PDF file of an unedited manuscript that has been accepted for publication. As a service to our customers we are providing this early version of the manuscript. The manuscript will undergo copyediting, typesetting, and review of the resulting galley proof before it is published in its final citable form. Please note that during the production process errors may be discovered which could affect the content, and all legal disclaimers that apply to the journal pertain. 


\title{
Sperm-dependent parthenogens delay the spatial expansion of their sexual hosts
}

\author{
K. Janko ${ }^{1 *}$ J. Eisner ${ }^{2}$ \\ ${ }^{1}$ Laboratory of Fish Genetics, Institute of Animal Physiology and Genetics, \\ Academy of Sciences of the Czech Republic, \\ Rumburská 89, 27721 Liběchov, Czech Republic. \\ ${ }^{2}$ Department of Theoretical Ecology, Institute of Entomology, Biology Centre ASCR, \\ Branišovská 31, 37005 České Budějovice, Czech Republic.
}

July 31, 2009

\begin{abstract}
It has been known for long time that asexual organisms may affect the distribution of sexual taxa. In fact, such phenomenon is inherent in the concept of geographical parthenogenesis. On the other hand, it was generally hypothesized that sperm-dependent asexuals may not exercise the same effect on related sexual population, due to their dependence upon them as sperm-donors. Recently, however, it became clear that sperm-dependent asexuals may directly or indirectly affect the distribution of their sperm-hosts, but rather in a small scale. No study addressed the large-scale biogeographic effect of the coexistence of such asexuals with the sexual species. In our study we were interested in the effect of sexual-asexual coexistence on the speed of spatial expansion of the whole complex. We expand previously published Lotka-Volterra model of the coexistence of sexual and gynogenetic forms of spined loach (Cobitis; Teleostei) hybrid complex by diffusion. We show that presence of sperm-dependent parthenogens is likely to negatively affect the spatial expansion of sexuals, and hence the whole complex, compared to pure sexual population. Given that most of the known sperm-dependent asexual complexes are distributed in areas prone to climate-induced colonization/extinction events, we conclude that such mechanism may be an important agent in determining the biogeography of sexual taxa and therefore requires further attention including empirical tests.
\end{abstract}

Keywords: Sperm-dependent asexuality, Cobitis, spatial expansion, dispersal, postglacial recolonization, hybridization.

\footnotetext{
*Author for correspondence: Fax: +420 315 639510; E-mail:janko@iapg.cas.cz
} 
Sperm-dependent. TEX version 17-[July 31, 2009; 1296]

\section{Introduction}

More than a century after the recognition of the "paradox of sex" (Weismann 1889), there is little disagreement that strict asexuality among higher organisms generally represents an evolutionary dead-end due to the inability of clones to escape either mutation or parasite loads, or to cope with a changing environment (e.g. Dybdahl and Lively (1998), Paland et al. (2006), West et al. (1999)). It should however be noted that some findings of exceptional longevity of clones, their adaptive potential or even the reevolution of sex contrast traditional assumptions in some model groups (Vrijenhoek 1993, Judson et al. 1996, Domes et al. 2007, Normark et al. 2003, Martens et al. 2003).

Whether or not asexuals are looked at as short-lived offshoots of sex, there is one aspect of their existence that is not fully appreciated in the discussion as to whether asexual organisms should be considered no-hopers or hopeful monsters (Vrijenhoek 1989); their potential to affect the distributions of sexual species. Although cases where asexuality has completely replaced sex (i.e. where there is no sexual species closely related to clonal species) are rare among Metazoans, asexuals do interfere with their sexual competitors even without driving sexual species to extinction. Geographical parthenogenesis may at least in some cases be viewed as the occupation by asexuals of habitats where sex would have normally existed (e.g. Bierzychudek (1985), Peck et al. (1998), Kearney (2003)). Although the evolutionary potential of asexual organisms is traditionally studied in terms of their abilities to persist some number of generation without sex or the creation of some evolutionary novelties, we should perhaps focus on questions of what their presence means to competing sexual species. Even if a given clone eventually goes extinct, the changes that its presence induced in the sexual species-such as affected distribution ranges, ecological or behavioral adaptations, or mate recognition mechanisms (e.g.

Lynch (1984), Peck et al. (1998), Ryan et al. (1996), Gabor and Ryan (2001), Schlupp (2005)) may represent an evolutionary impact not generally considered.

Sperm-dependent (pseudogamous) asexuals are parthenogenetic organisms that require sperm to activate the oocyte development, and are thus dependent on a suitable sperm donor in what could be considered a kind of parasitism (see e.g. Schlupp (2005), Choleva et al. (2008) and citations therein). It has been speculated for decades (see. e.g. Moore and McKay (1971)) that all else being equal, the sperm-dependent asexuals should out-compete their hosts and the whole complex collapse. Therefore, various mechanisms have been proposed to ensure the coexistence of both populations. For example, sexual males are likely to evolve mate-recognition since the sperm used for the activation of clonal ova is effectively wasted. This may be manifested either by the premating choice of partner (Ryan et al. 1996, Gabor and Ryan 2001) or by differential allocation of sperm (Schlupp and Plath 2005). In asexuals, selection among clones operates to 
overcome the mating preference for conspecifics in the sexual species by more aggressive mating behavior or by the evolution of sex-mimicry (Beukeboom and Vrijenhoek 1998). Competition for resources with the parental species may select the asexual population to either minimize the niche overlap by specializing to alternative habitats (Hellriegel and Reyer 2000, Plenet et al. 2005) or to form narrowly specialized clonal lineages able to outcompete the sexual host in given sub-niches and therefore to coexist in the same habitat (Vrijenhoek 1979, Pound et al. 2002). Parasites may also preferentially affect asexuals, because clonal reproduction does not generate enough variability to escape them (e.g. Dybdahl and Lively (1998), Hakoyama and Iwasa (2004)).

Aspects of spatial segregation of sexual and sperm-dependent asexual components have rarely been taken into account, but if so, the studies suggested importance of such aspects for the long-term persistence of such mixed complexes. These works either assumed the twodeme model (Hellriegel and Reyer 2000), or searched for dispersal-extinction equilibria in a metapopulation framework (e.g. Kokko et al. (2008)). Interestingly, Kokko et al. (2008) showed that metapopulation dynamics alone may promote stable coexistence. This is expected either if the sperm-parasite causes little damage to its host (thus resulting in majority of demes being occupied by both species), or if the damage is rather high, locally driving the host population quickly to extinction (thus resulting in a relatively low invasion rate of the parasite).

Both abovementioned studies assumed population dynamics in a constantly inhabited environment, because the sperm-dependent parthenogens are usually expected to have only a small effect on the large-scale biogeography of the sexual taxa on which they critically depend. Sperm dependent asexuals are generally assumed to arise in areas where their sexual progenitors are present and subsequently invade their ranges without driving sexual hosts to extinction. However, several sperm-dependent asexual 'species' are known to have either abandoned their parental host species or out-competed it with a subsequent switch to another sperm-host (rev. in Choleva et al. (2008)), resulting in a type of geographical parthenogenesis even in the case of the sperm-dependent asexuality. While such effects of sperm-dependent asexuals are rare, they may possibly influence the large-scale biogeography of sexual species yet by a different, possibly quite frequent, process; they may attenuate the rate of spatial spread of their sexual hosts.

We noticed such a possibility when reconstructing the phylogeography of the Cobitis taenia hybrid complex. Here, six parapatric species, i.e. C. elongatoides, C. taenia, C. tanaitica, C. taurica, C. strumicae and C. melanoleuca, are known to hybridise producing several virtually all-female hybrid forms that coexist with the parental species and reproduce by gynogenesis (a type of sperm-dependent asexuality, where the sperm triggers the oocyte development, but does not contribute to genetic build-up of progeny; reviewed in Janko et al. (2007a)). Such 
Sperm-dependent. TEX version 17-[July 31, 2009; 1296]

hybridizations likely take place during interglacial periods, when sexual species intermittently come into reproductive contact and the resulting gynogenetic hybrid all-female lineages subsequently invade the areas of parental species (Janko et al. 2005). Two of the parental species, $C$. taenia and C. elongatoides, are widespread in Europe, but whereas the former species inhabits most of the eastern, western and northern Europe, the latter is restricted to the Danubian watershed and to upper stretches of the Odra and Elbe rives. Culling et al. (2006) showed that the current distribution of $C$. taenia including western Europe, is a result of recent, probably post-glacial, colonization from the east.

It is not clear why in the case of Cobitis the Danube did not play an important role in the colonization of Europe, in contrast to other cases of freshwater fish (e.g. Durand et al. (1999), Nesbø et al. (1999), Kotlík and Berrebi (2001), Bohlen et al. (2006)). This is especially striking, as $C$. elongatoides was apparently able to expand into Central Europe, but not any further. This problem has many more or less plausible explanations, but our previous analysis (Janko et al. 2005) suggests that C. taenia expanded as a 'pure' sexual population, which was 'infected' by gynogenetic asexuals only after the postglacial reproductive contact with $C$. elongatoides expanding from the Danubian refuge. On the other hand, C. elongatoides expansion was about twice as slow as C. taenia and was probably coupled with the co-expansion of an ancient elongatoides-tanaitica gynogenetic hybrid lineage that survived glacial events in the Danubian refuge. Since asexuals compete with host species for resources and sperm, thereby decreasing population densities, we speculated that they may also reduce the dispersal potential of the sexual population. We proposed it as the reason why the Cobitis colonization of Western Europe came from the east rather than from the Danube.

In this paper we study this hypothesis by expanding published Lotka-Volterra model describing the local dynamics of Cobitis hybrid complex by adding a linear diffusion. Since this model assumed no differentiation between sexual and asexual forms, we also expanded model of Schley et al. (2004) describing the coexistence of sexual and sperm-dependent asexual hermaphroditic populations that may differ either in competitive ability or intrinsic capacity to exploit resources. We stress on the choice of parameters under which both population coexist (and not to perform a full bifurcation analysis of our models which could be rather complex) and show that presence of sperm-dependent parthenogens is likely to negatively affect the spatial expansion of the whole complex under some circumstances. Given that ranges of many of the known sperm-dependent asexual complexes likely underwent repeated extinction/expansion cycles, we suggest that such a mechanism may be an important agent in determining the biogeography of sexual taxa. 
Sperm-dependent. TEX version 17-[July 31, 2009; 1296]

\section{Methods}

\section{Equations}

The Lotka-Volterra model has been previously used to describe local coexistence of spermdependent parthenogens with their sexual hosts (e.g. Schley et al. (2004), Pound et al. (2002)).

In this article, we first took advantage of Bobyrev et al. (2003)-model of three ordinary differential equations describing the temporal behavior of Cobitis taenia hybrid complex

$$
\begin{aligned}
& \frac{\mathrm{d} R}{\mathrm{~d} t}=r R(1-R / K)-\alpha_{B} B R-\alpha_{U} U R, \\
& \frac{\mathrm{d} B}{\mathrm{~d} t}=\gamma_{B} \alpha_{B} B R-\mu_{B} B, \\
& \frac{\mathrm{d} U}{\mathrm{~d} t}=\gamma_{U} \alpha_{U} U R\left(1-e^{-S B}\right)-\mu_{U} U,
\end{aligned}
$$

where the first equation describes the dynamics of the resource population, $R$, which is assumed to grow logistically in the absence of consumers ( $r$ is a Malthusian parameter, $K$ is the environment capacity). For simplicity, the functional response of the sexual $(B)$ and asexual $(U)$ forms is assumed to be proportional, with $\alpha_{B}$ and $\alpha_{U}$ being the parameters of search efficiency, respectively. The second and the third equations reproduce the dynamics of the sexual species, $B$, and the asexual form, $U$, in terms of their abundances. Both populations exponentially decay without food with mortality coefficients $\mu_{B}$ and $\mu_{U}$. Notations $\gamma_{B}$ and $\gamma_{U}$ stand for coefficient of food conversion into offspring for the sexual and the asexual form, respectively. The term $1-e^{-S B}$ in the third equation is traditionally used for description of the Allee effect in polygynous populations (McCarthy 1997) and determines the dependence of the asexual population growth rate on the availability of males from the sexual population.

This model has two conditions for a non-trivial equilibrium existence, one depending strictly on the ratios of $\alpha, \gamma$ and $\mu$ parameters in sexuals and asexuals, respectively, which allowed authors to empirical test whether natural observations support such a stable coexistence.

However, Bobyrev et al. (2003) system did not assume any Allee effect on sexual host, which we consider as a drawback. To alleviate such simplification, we modified Bobyrev et al.'s equations according to published formulas of Schley et al. (2004), keeping in mind that our system incorporates a sexual host population (where males usually constitute about a half of the sexual population size), whereas Schley et al. modelled a population of hermaphrodites. When these variations are applied, the equations expand to incorporate the spatial variable:

$$
\begin{aligned}
\frac{\partial R}{\partial t} & =d_{R} \frac{\partial^{2} R}{\partial x^{2}}+r R(1-R / K)-\alpha_{B} B R-\alpha_{U} U R, \\
\frac{\partial B}{\partial t} & =d_{B} \frac{\partial^{2} B}{\partial x^{2}}+\gamma_{B} \alpha_{B} R \frac{B / 2}{B+U} B / 2-\mu_{B} B, \\
\frac{\partial U}{\partial t} & =d_{U} \frac{\partial^{2} U}{\partial x^{2}}+\gamma_{U} \alpha_{U} R \frac{B / 2}{B+U} U-\mu_{U} U,
\end{aligned}
$$


Thus the reproductive success of the whole complex depends - in part - on the population of males of the sexual species. Assuming that the individuals of both populations choose a mate at random from the entire population we modelled such dependence by the term $(B / 2) /(B+U)$, which is just the Allee effect in the formula used in (Schley et al. 2004). We put the abundances of males and females in sexual population as equal to $B / 2$. The higher the proportion of parthenogens, the fewer the proportion of males available to fertilize the eggs of both sexual and parthenogenetic females. Just as Bobyrev et al. (2003) suggested for Cobitis taenia hybrid complex, we assumed no niche segregation between both populations. Moreover, we set the reproduction of sexual diploids proportional to the number of females $(B / 2)$.

Second model we will stress on is the system (3) from Schley et al. (2004), again supplemented by a linear diffusion:

$$
\begin{aligned}
& \frac{\partial B}{\partial t}=d_{B} \frac{\partial^{2} B}{\partial x^{2}}+\left(\frac{B}{B+\kappa U}-\mu-\left(B+\kappa \eta_{U} U\right)\right) B \\
& \frac{\partial U}{\partial t}=d_{U} \frac{\partial^{2} U}{\partial x^{2}}+\left(\frac{\beta B}{B+\kappa U}-\mu-\left(\frac{\eta_{B}}{\kappa} B+U\right)\right) U .
\end{aligned}
$$

Here, $\mu$ is the death rate, $\eta_{U}$ and $\eta_{B}$ measures the inhibitory effect per capita of type $U$ and $B$ on reproduction by type $B$ and $U$ relative to the intraspecific impact on reproduction of type $B$ and $U$, respectively. The coefficient $\beta$ is the intrinsic reproductive rate per capita of asexual triploids relative to sexual diploids, $\kappa$ represents the proportional increase in the ability of asexuals to exploit their environment, relative to that of sexuals.

\section{The model}

Although spatial expansion of biological systems including current asexual complexes involves more than one dimension, we present a simplifying assumption of expansion in one-dimensional space to reduce the complexity of computation, which is likely to capture the key features (Murray 2002, p.439). We used a numerical approach to find a solution of the above system of differential equations.

We have considered zero flux (i.e. Neumann) boundary conditions for all variables. Initially for $t=0$ we set Resource to its carrying capacity $K$ in the whole domain under consideration, sexuals and asexuals were set to (an arbitrarily chosen) value $U_{0}=B_{0}=10^{-4}$ only on the left end of the domain while they were considered absent inside the domain. (The sensitivity on the initial conditions will be discussed below.) For the numerical simulations we took for the domain an interval of the length $\ell=160$ and we divided it onto $n=60$ discrete pieces (the values were chosen to produce well-resolved solutions). We have compared two simulation runs of the program for both models: first with sexuals present and second without them. We defined zero initial values for $U$ and $B$ with the exception of the first discrete node where 
Sperm-dependent. TEX version 17-[July 31, 2009; 1296]

both $U$ and $B$ in the first run and $B$ only in the second run were set to the initial value $10^{-4}$. Diffusion coefficients $d_{B}$ and $d_{U}$ of fish populations were set to 0.1 , Resource was assumed to disperse more slowly $\left(d_{R}=0.001\right)$. Choosing a more fine mesh of our interval would mean only a rescaling of the diffusion coefficients/of the interval under consideration and would not give us better/more realistic results. Since we do not know the realistic values of diffusion coefficients, we fixed the above mentioned values.

\section{Parameters}

Let us recall that our task was to find particular parameters for both models for which both sexual and asexual populations can coexist and to show that 1) they co-expand under such circumstances and 2) the speed of expansion of the hybrid complex is lower than that of purely sexual population.

In the numerical analysis of the model (2), we first set all parameters equal for sexuals and asexuals and varied parameters $r$ and $K$ of the resource. To study the influence of the parameters corresponding to $B$ and $U$ we subsequently varied their $B / U$ ratios (keeping the values of remaining parameters for sexuals and asexuals equal). In particular, we adjusted the $B / U$ ratios of $\alpha, \mu$ and $\gamma$ so that neither sexuals nor parthenogens would go extinct. Conditions implying stable coexistence of sexual and asexual competitors in general and sperm-dependent parthenogens in particular, have been extensively studied elsewhere (see the Introduction) and are not the subject of this study. Hence, our simplification is reasonable.

Since every model, including the present one, depicts only a part of the reality, it is important to test its predictions on real-world examples. Therefore, in a second set of analyzes, we used the empirically estimated ratios of the parameters by Bobyrev et al. (2003) on a mixed diploid-polyploid population of spined loaches from the Moscow River. In this case, triploids

were composed entirely by gynogenetic females, while diploids were uniquely sexual females and males serving as host population. The estimated parameter ratios were: $\mu_{B} / \mu_{U}=1.08$, $\alpha_{B} / \alpha_{U}=0.81$ and $\gamma_{B} / \gamma_{U}=1.12$.

In the numerical analysis of the model (3) we followed the analysis performed in their paper (Schley et al. 2004), i.e. we have varied successively the parameters $e t a_{U}, \kappa$ and $\beta$ (each run only one parameter), other parameters were fixed. The last run was with all parameters equal.

\section{Results}

The results of numerical simulations are demonstrated on the figures. To demonstrate the influence of a particular parameter we varied one parameter for each figure only keeping the 
Sperm-dependent. TEX version 17-[July 31, 2009; 1296]

other ones fixed. To allow the comparison between figures, fixed parameters were kept on the same values.

On each particular figure a spatial occupation of sexuals (in upper half) and of the sexuals/asexuals complex (in lower half of each figure; sexuals on the left, asexuals on the right) is shown. To make the figures clear, sexual and asexual populations are drawn separately but in fact, they invade simultaneously from the same initial (left) end of the interval under consideration and occupy the same space.

\section{Spatial expansion under modified Bobyrev model}

First we set all parameters for $B$ and $U$ equal. Since model (2) assumes strictly two-fold advantage of asexuality, asexuals, not surprisingly, outcompeted their sperm donors and whole system collapsed after a short-term spatial expansion resulting from initial introduction of both consumers into the first discrete node (Fig. 1A).

To ensure the coexistence and co-expansion of both populations when dispersal coefficients are equal, either the fitness of parthenogens had to be decreased or the fitness of sexuals had to be increased. This was achieved either by increasing $\alpha_{B}$ and/or $\gamma_{B}$ or by decreasing $\mu_{B}$.

We performed the same simulations also with different initial seed values for sexual and asexual populations (relative initial proportion of sexuals to asexuals varied from 0.1 to 100). We obtained the same qualitative results with regards to the expansion attenuation, but the surviving complex occupied the area in different densities. The larger was the ratio of initial values for $B$ to $U$ the larger density was established and the smaller $K$ was necessary to support both populations.

For low values of the $\alpha_{B}$ all populations went to extinction (because the value of $K$ was too low to support even pure sexual population) (Fig. 2A). When $\alpha_{B}$ was larger than 1.2 (i.e. when $\alpha_{B} / \alpha_{U}$ was larger than 0.8) only pure sexual population was able to expand but asexuals out-competed their hosts in the mixed populations leading to a collapse (Fig. 2B). For $\alpha_{B} / \alpha_{U}$ approaching values of 2 , competing asexuals apparently affected the density of sexual population behind the front line of its expansion. This effect was manifested by the slower tempo of sexuals' spatial spread compared to a purely sexual population and was the strongest when $\alpha_{B} / \alpha_{U}$ equaled 2 (Fig. 2C). Above the value 2.1, we observed the spatial expansion of asexuals, but their expansion was more and more delayed behind the sexual one and in lower and lower densities with the simultaneously loosing the influence on the expansion speed of sexuals (Fig. 2D).

We observed similar behavior by increasing the $\gamma_{B} / \gamma_{U}$ ratio (Fig. 3A-D).

The effect of decreasing mortality of sexuals was similar. When the $\mu_{B} / \mu_{U}$ ratio was less than about 0.2, asexuals followed the expansion wave of their sperm donors, but went 
Sperm-dependent. TEX version 17-[July 31, 2009; 1296]

extinct in the interior zone behind the expanding front due to the depletion of resources by consumers (Fig. 4A). This means that the underlying dynamics of the steady system leads to extinction of asexuals but the population survives along a travelling wave. Even in such a case, however, the co-expansion of asexuals slowed down the spread of the whole complex compared to the expansion speed of the pure sexual population. When this ratio increased we observed a co-expansion of both populations with an attenuating effect of asexuals on the speed of the expansion of the whole complex (Fig. 4B). This effect was stronger with increasing $\mu_{B}$ (Fig. 4C). With $\mu_{B} / \mu_{U}$ above 0.55 , asexuals were too strong competitors to allow for coexistence with a sexual population (Fig. 4D).

Also the environmental carrying capacity had strong influence on the final outcome of both scenarios (i.e. pure sexual and mixed sexual-asexual). To study its effect, we set $\alpha_{B} / \alpha_{U}=2$, $\gamma_{U} / \gamma_{B}=1$ and $\mu_{B} / \mu_{U}=1$. Under very low $K$ neither population was able to survive (Fig. $5 \mathrm{~A}$ ). Only when $K$ increased, the expansion started (Fig. 5B). We observed positive correlation between the value of $K$ and the speed of propagation of both types of consumers (Fig. 5AD). This is an expected behavior, since the linear operator of second derivatives causes the dependence of the speed of the expansion on the population densities and the speed of the traveling wave front is known to depend on the square root of the growth rate and diffusion coefficient (Murray 2003).

The immediate effect of asexuals was that more than 2 times higher $K$ (2.5 versus 1.2) was needed to allow for persistence and expansion of the mixed population compared to the pure sexual one (Fig. 5B,C). Such patterns are intuitive given that part of the resources must be consumed by asexuals or else this wouldn't be a mixed population. This effect results in lower abundances of sexuals compared to the pure population for the same value of $K$. The presence of asexuals always slowed down the expansion speed compared to the expansion rate of the pure population at the same value of $K$.

Under empirically estimated $B / U$ parameter ratios (Bobyrev et al. 2003), asexuals always outcompeted the sperm-donors and the whole population collapsed.

\section{Spatial expansion under Schley model}

Setting all parameters equal we observed the expansion of all populations with slowing down in the hybrid complex (Fig. 1B).

The effect of increasing $\kappa$ was similar to that of increasing $\mu_{B} / \mu_{U}$ in the Bobyrev model. When $\kappa$ was less than about 0.9 , asexuals survived along a travelling wave but went extinct in the interior zone behind the expanding front (Fig. 6A). Nevertheless, the co-expansion of asexuals slowed down the spread of the whole complex compared to the expansion speed of the pure sexual population. When $\kappa$ increased up to one we observed a co-expansion of both 
Sperm-dependent. TEX version 17-[July 31, 2009; 1296]

populations with an attenuating effect of asexuals on the speed of the expansion of the whole complex (Fig. 1B). Further increasing of $\kappa$ lead to the co-expansion of both populations but their density started to be lower and lower in the interior of the occupied space (similarly as the asexuals did for small $\kappa$, Fig. 6B,C). Simultaneously, the expansion was slower and slower. For even larger $\kappa$ (more than 25), the asexuals started to be strong competitors and they out-competed the sexual population (Fig. 6D). Simultaneously, the larger $\kappa$ was, the lower population densities were observed.

Next we studied the effect of the competitive impact of sexuals onto asexuals under the very low mortality rate $\mu$ (Schley et al. 2004, Subsection 3.1.1). If the direct impact of asexuals onto sexuals was small $\left(\eta_{U}<1\right)$, both populations co-expand and sexuals were slowed down by asexuals (Fig. 7A) since they influenced the sexuals via nontrivial $\kappa$ (we set $\kappa=1$ as Schley did). For $\eta_{U}>1$, the direct influence became strong leading to the decreasing population density of both populations in the occupied area. This effect was more visible in the sexual population because their travelling wave formed stronger peak than that of asexuals (Fig. 7B). This effect positively correlated with $\eta_{U}$. The populations balanced on the lower and lower values but still survived and expand (Fig. 7C).

The subject of the last runs is the influence of the intrinsic reproductive rate per capita of asexuals relative that to sexuals. For low values of the parameter $\beta$ the asexual population went extinct after a short period of expansion with almost no effect on their sexual hosts (Fig. 8A). For $\beta$ approaching one we observed co-expansion with slowing effect of asexuals (Fig. 8B). This effect positively correlated with increasing values of $\beta$ (Fig. $8 \mathrm{C}$ ) until high values when asexuals started to out-compete the sexual population. Densities of both expanding populations negatively correlated with $\beta$.

\section{Discussion}

Previously published studies of the spatial aspects of sperm-dependent parthenogenesis (Hellriegel and Reyer 2000, Kokko et al. 2008) provided very innovative insights into the role of the space in maintenance of sperm-dependent asexuality. However, given that distribution ranges of many species have frequently undergone shifts in relations to climate changes (Hewitt 2000), it is instructive to study the role of sperm-dependent parasites in the process of recolonization of newly available areas.

Present extensions of both previously published models of pseudogamous asexual complex show that coexisting sperm-dependent parthenogens tends to attenuate the speed of spatial propagation of the whole complex.

We must stress that application of both models is simplistic. We did not take into account 
Sperm-dependent. TEX version 17-[July 31, 2009; 1296]

various parameters, which may also affect the coexistence of sperm-dependent asexuals and their sexual hosts. This could be e.g. mate choice (Hellriegel and Reyer 2000, Ryan et al. 1996, Gabor and Ryan 2001, Schlupp and Plath 2005), or the role of parasites preferentially attacking the asexuals (Hakoyama and Iwasa 2004). On the other hand, abovementioned theoretical studies treated the problem of coexistence of host and sperm-parasite in detail, including the work of Schley et al. (2004) dealing with effects of differences in competitive or resource exploitation abilities. While relative importance of such processes may vary from case to case, our interest in general effect of coexistence between sexuals and sperm-dependent asexuals on spatial expansion of the whole complex justifies the ad hoc choice of parameter values.

Indeed, the slow-down of the population expansion speed in response to the competition has been demonstrated previously using reaction-diffusion models (e.g. Hart and Gardner (1997), Skalski (2004)). For example, the minimal speed of the expansion in the two-species Lotka-Volterra competition model has been derived as

$$
C_{\min }=2 \sqrt{\alpha_{1} D_{1}\left(1-c_{1} K_{2}\right)}
$$

where $\alpha_{1}$ and $D_{1}$ are the intrinsic growth rate and diffusion coefficient of the invader, $c_{1}$ is the competition effect of species 2 on species 1 and $K_{2}$ is the carrying capacity of the species 2 (see Murray (2003)). Our findings, which show the attenuating effect of coexisting sperm-dependent parasite asexuals on spatial expansion of host population, are therefore well in agreement with predictions.

The phenomenon of geographical parthenogenesis implies that the presence of asexuals affects the distribution of competing sexual species (see Peck et al. (1998) for theoretical treatment of the problem and Kearney (2005) for discussion of empirical examples). Kokko et al. (2008) further studied the conditions under which sperm-dependent asexuals may affect the spatial distribution of their hosts. Here, we demonstrate two additional effects: first, depending on the environment, they may either delay, or even stop the onset of the expansion of the host population and second, they may slow down the speed of population expansion. As in previous works applying the reaction-diffusion models (e.g. Murray (2003), Skalski (2004)), we analyzed the expansion continuous in space and time, which was initiated from single population, while in nature the colonization of new habitat may proceed in more discrete steps from several sources. As predicted by Schley et al. (2004) and demonstrated by Kokko et al. (2008), spatial dynamics of pseudogamous asexual complex in constantly inhabited space is likely approximated by metapopulation model with repeated extinctions/recolonizations of patches by either sexual or asexual component. The priority effects stemming from such stochastic events may be important of course. If the spatial expansion is initiated by asexual-free deme, sexuals may expand at rate, which is unaffected by asexuals lagging behind. Nevertheless, keeping 
Sperm-dependent. TEX version 17-[July 31, 2009; 1296]

in mind the necessary simplifications of our model, this study points at previously unnoticed role for sperm-dependent asexuals; they may be decisive elements in shaping bio-geographical patterns over large areas.

Many pseudogamous complexes are of hybrid origin involving two or more hybridizing sexual species and occur in areas, which are prone to climate-induced shifts in distribution ranges (rev in Beukeboom and Vrijenhoek (1998), Schlupp (2005). Contemporary distributions of clones and parental species is therefore supposed to mirror different ecological requirements of parental species as well as their routes and rates of interglacial expansions (e.g. Berger (1977), Angers and Schlosser (2007)). Our study indicates that absence of particular parental species over large parts of distribution range of the clonal complex does not necessarily reflect low colonization abilities or different ecological adaptations, but may result from the fact that coexpanding clones have attenuated its expansion from glacial refugia.

For example, Janko et al. (2005) showed that gynogenetic lineages of the C. taenia asexual complex arose on multiple occasions, when parental species repeatedly came into contact during interglacial periods. One of those lineages was able to survive several glacial maxims in a Danubian refuge and co-expanded with $C$. elongatoides to Central Europe during the Holocene. While it is possible that without co-expanding gynogens, $C$. elongatoides would have been able to colonize the rest of the Continent like other Danubian fish species, gynogens putatively attenuated its expansion rate, which might have given an opportunity to C. taenia expanding from the East to settle there first.

We recognize that to test this hypothesis on real examples, more detailed models should be applied and more field data must be available for any given complex. In the Cobitis example, simulations under empirical parameter estimates of Bobyrev et al. (2003) resulted in a demise of the whole complex due to the out competition of sexuals by sperm-dependent asexuals. This is rather implausible result since we know that both forms successfully coexist in nature. Such a failure may result from the fact that present model did not incorporate some of the abovementioned behavioral or ecological mechanisms affecting the persistence of asexual complex. In fact, when performing the analysis with original equations from Bobyrev et al. (2003), we noticed that the parameter $S=m \cdot d$ strongly affects the outcome, but unfortunately, the authors never explained how $S$ was estimated and what value was used for numerical solution. It is also possible that $U / B$ ratio of parameters estimated by Bobyrev et al. may be partly misleading. For example, the estimates of $\gamma$ were based on the number of eggs per female, but authors did not take into account possible differences in hatching success between diploid sexual and triploid asexual females as well as the fact that significant proportion of triploid eggs may be wasted due to the incorporation of sperms genome (Janko et al. 2007b) Such processes are likely to decrease the $\gamma_{U} / \gamma_{B}$ ratio. The situation seems similar in other sperm-dependent complexes. 
Sperm-dependent. TEX version 17-[July 31, 2009; 1296]

In one of the best known example, the dynamics of coexistence of sexual and asexual forms of water frog complex was described with an impressive amount of biologically relevant traits (including local migration; Hellriegel and Reyer (2000)), but incomplete knowledge of some of the parameter values would prevent clear-cut model based inference of expansion history.

Direct testing of the hypothesis that sperm-dependent asexuals delay the spatial expansion of their sperm donors would require the inclusion of major biologically relevant traits into the model as well as their empirically estimated values, but so far, the incomplete knowledge about ecology, behavior and, mainly, the dispersal rates in most of such complexes prevents us from more advanced parameter estimates and formulation of more complex models. Therefore, rather than drawing definitive conclusions about the role of sperm-dependent asexuals in the biogeography of sexual taxa, our intention is to point at the existence of such a previously unrecognized mechanism, which may be important aspect in the evolution of sperm-dependent asexual complexes.

\section{Acknowledgement}

Grant Agency of the Czech Republic No. GAČR 206/05/P586 and No. GAČR 206/06/1763 provided the support for K. J.. The Laboratory of Fish Genetics at Liběchov receives continuous support from the Academy of Sciences of the Czech Republic (IRP IIAPG No. AV0Z50450515). J. E. has been supported by the grant KJB600070602 of the Grant Agency of the Academy of Sciences of the Czech Republic.

\section{References}

Angers, B., Schlosser, I. J., 2007. The origin of Phoxinus eos-neogaeus unisexual hybrids. Molecular Ecology 16, 4562-4571.

Berger, L., (1977). Systematics and hybridization in the Rana esculenta complex, Pages 367388 in H. D. Taylor and I. S. Guttman, eds. The reproductive biology of amphibians, Plenum Press, New York and London, pp. 367-388.

Beukeboom, L. W., Vrijenhoek, R. C., 1998. Evolutionary genetics and ecology of spermdependent parthenogenesis. J. Evol. Biol. 11, 755-782.

Bierzychudek, P., 1985. Patterns in plant parthenogenesis. Experientia 41, 12551263.

Bobyrev, A., Burmensky, V., Vasil'ev, V., Kriksunov, E., Lebedeva, E., 2003. Coexistence of triploid and diploid forms of spined loach, Cobitis taenia: a model-based approach. Folia Biologica-Krakow, Suppl. S 51, 55-60. 
Sperm-dependent. TEX version 17-[July 31, 2009; 1296]

Bohlen, J., Šlechtová, V., Bogutskaya, N., Freyhof, J., 2006. Across Siberia and over Europe: Phylogenetic relationships of the freshwater fish genus Rhodeus in Europe and the phylogenetic position of $R$. sericeus from the River Amur. Molecular Phylogenetics and Evolution $40,856-865$.

Choleva, L., Apostolou, A., Rab, P., Janko, K., 2008. Making it on their own: sperm-dependent hybrid loaches (Cobitis; Teleostei) switch the sexual hosts and expand beyond the ranges of their original sperm-donors. Philosophical Transactions of the Royal Society 363, 29112919.

Culling, M., Janko, K., Boroń, A., Vasil'ev, V., Coté, I., Hewitt, G., 2006. European Colonisation by the Spined Loach Cobitis taenia from Ponto-Caspian Refugia based on mitochondrial DNA variation. Molecular Ecology 15, 173-190.

Domes, K., Norton, R., Maraun, M., Scheu, S., 2007. Reevolution of sexuality breaks Dollo's law. Proc. Natl. Acad. Sci. USA 104, 7139-7144.

Durand, J., Persat, H., Bouvet, Y., 1999. Phylogeography and postglacial dispersion of the chub (Leuciscus cephalus) in Europe. Molecular Ecology 8, 989-997.

Dybdahl, M., Lively, C., 1998. Host-parasite coevolution: Evidence for rare advantage and time-lagged selection in a natural population. Evolution 52, 1057-1-66.

Gabor, C., Ryan, M., 2001. Geographical variation in reproductive character displacement in mate choice by male Sailfin Mollies. Proc. R. Soc. B 268, 1063-1070.

Hakoyama, H., Iwasa, Y., 2004. Coexistence of a sexual and a unisexual form stabilized by parasites. Journal of Theoretical Biology 226, 185-194.

Hart, D., Gardner, R., 1997. A spatial model for the spread of invading organisms subject to competition. J. Math. Biol. 35, 935-948.

Hellriegel, B., Reyer, H., 2000. Factors influencing the composition of mixed populations of a hemiclonal hybrid and its sexual host. J. Evol. Biol. 13, 906-918.

Hewitt, G., 2000. The genetic legacy of the Quaternary ice ages. . Nature 405, 907-913.

Janko, K., Bohlen, J., Choleva, L., Šlechtová, V., Flajšhans, M., Rábová, M., Ráb, P., Šlechta, V., Vassileva, E., Vassilev, V., Ivanova, P., Dobrovolov, P., Nalbant, T., Kontula, T., Culling, M., Persat, H., Kotusz, J., Kullander, S., 2007a. Species- and hybrid richness in spined loaches (genus Cobitis L.) II.: up-to-date overwiev of geographic distribution of 
Sperm-dependent. TEX version 17-[July 31, 2009; 1296]

sexual and clonal forms of the o called C. taenia hybrid complex. Journal of Fish Biology 71 (Supplement X), 1-22.

Janko, K., Bohlen, J., Šlechtová, V., Flajšhans, M., Lamatch, D., Kotlí, P., Ráb, P., $2007 b$. Evidence for gynogenesis as the reproductive mode of hybrid loaches (Cobitis: Teleostei) and the implications for the evolution of polyploidy. Genetica 131, 185-94.

Janko, K., Kotlík, P., Culling, M., Ráb, P., 2005. Ice age cloning - comparison of the Quaternary evolutionary histories of sexual and clonal forms of spiny loaches (Cobitis; Teleostei) using the analysis of mitochondrial DNA variation. Molecular Ecology 14, 2991-3004.

Judson, O., , Normark, B., 1996. Ancient asexual scandals. Trends Ecol. Evol. 11, 41-46.

Kearney, M., 2003. Why is sex so unpopular in the Australian desert? Trends in Ecology and Evolution 18, 605-607.

Kearney, M., 2005. Hybridization, glaciation and geographical parthenogenesis. Trends in Ecology and Evolution 20, 495-502.

Kokko, H., Heubell, K., Rankin, D., 2008. How populations persist when asexuality requires sex: the spatial dynamics of coping with sperm parasites. Proc. R. Soc. B 275, 817-825.

Kotlík, P., Berrebi, P., 2001. Phylogeography of the barbel (Barbus barbus) assessed by mitochondrial DNA variation. Molecular Ecology 10, 2177-2185.

Lynch, M., 1984. Destabilizing hybridization, general-purpose genotypes and geographic parthenogenesis. Quart. Rev. Biol. 59, 257-290.

Martens, K., Rossetti, G., Horne, D. J., 2003. How ancient are ancient asexuals? Proc. R. Soc. Lond. B Biol. Sci. 270, 723-729.

McCarthy, M., 1997. The Allee effect, finding mates and theoretical models. Ecol. Modell. 103, 99-102.

Moore, W., McKay, F., 1971. Coexistence in unisexualbisexual species complexes of Poeciliopsis (Pisces: Poeciliidae). Ecology 52, 791-799.

Murray, J., 2002. Mathematical Biology: I. An Introduction. Springer-Verlag, New York, USA.

Murray, J., 2003. Mathematical Biology: II. Spatial Models and Biomedical Applications. Springer-Verlag, New York, USA. 
Sperm-dependent. TEX version 17-[July 31, 2009; 1296]

Nesbø, C., Fosshein, T., Vøllestad, L., Jakobsen, K., 1999. Genetic divergence and phylogeographic relationships among European perch (Perca fluviatilis) populations reflect glacial refugia and postglacial colonization. Molecular Ecology 8, 1387-1404.

Normark, B., Judson, O., Moran, N., 2003. Genomic signatures of ancient asexual lineages. Biol. J. Linn. Soc. 79, 69-84.

Paland, S., , Lynch, M., 2006. Transitions to asexuality result in excess amino-acid substitutions. Science 311, 990-992.

Peck, J., Yearsley, J., Waxman, D., 1998. Explaining the geographic distributions of sexual and asexual populations. Nature 391 391, 889-892.

Plenet, S., Joly, P., Hervant, F., Fromont, E., Grolet, O., 2005. Are hybridogenetic complexes structured by habitat in water frogs? J. Evol. Biol. 18, 15751586.

Pound, G., Doncaster, C., Cox, S., 2002. A Lotka-Volterra Model of Coexistence between a Sexual Population and Multiple Asexual Clones J. J. Theor. Biol. 217, 535-545.

Ryan, M. J., Dries, L. A., Batra, P., Hillis, D. M., 1996. Male mate preferences in a gynogenetic species complex of Amazon mollies. Anim. Behav. 52, 1225-1236.

Schley, D., Doncaster, C., Sluckin, T., 2004. Population models of sperm-dependent parthenogenesis. J. Theor. Biol. 229, 559-572.

Schlupp, I., 2005. The Evolutionary Ecology of Gynogenesis. Annual Reviews of Ecology. Evolution and Systematics 36, 399-417.

Schlupp, I., Plath, M., 2005. Male mate choice and sperm allocation in a sexual/asexual mating complex of Poecilia (Poeciliidae; Teleostei). Biol. Lett. 1, 169-171.

Skalski, G. T., 2004. The diffusive spread of alleles in heterogeneous populations. Evolution $58,470-478$.

Vrijenhoek, R. C., 1979. Factors affecting clonal diversity and coexistence. Am. Zool. 19, $787-797$.

Vrijenhoek, R. C., (1989). Genetic and evolutionary constraints on the origin and establishment of unisexual vertebrates, Pages 24-31 in R. Dawley and J. Bogart, eds. Evolution and ecology of unisexual vertebrates, Vol. 466 of Bulletin, New York State Museum, Albany, New York., pp. 24-31. 
Sperm-dependent. TEX version 17-[July 31, 2009; 1296]

Vrijenhoek, R. C., 1993. The Origin and Evolution of Clones Versus the Maintenance of Sex in Poeciliopsis. J. Hered. 84, 388-395.

Weismann, A., 1889. Essays on heredity and kindred biological subjects. Oxford Univ. Press, Oxford, UK.

West, S., Lively, C., Read, A., 1999. A pluralist approach to sex and recombination. J. Evol. Biol. 12, 1003-1012.

A)

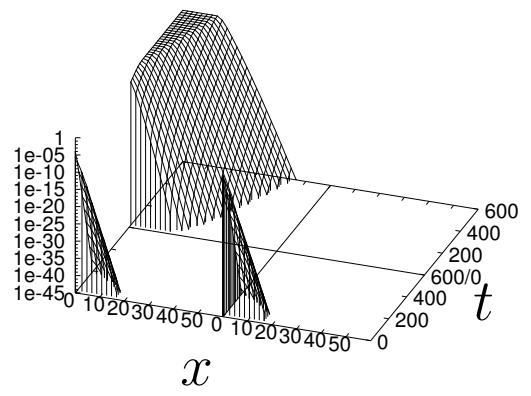

B)

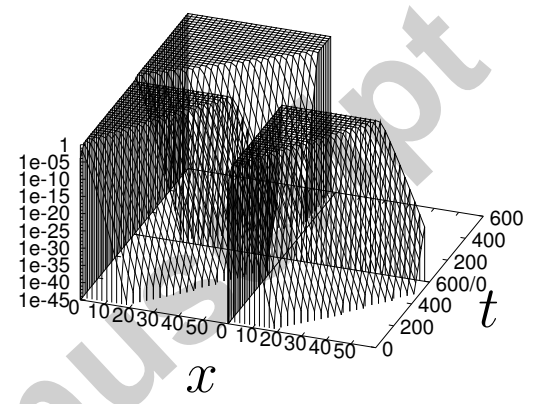

Figure 1: Spatial occupation of sexuals (upper half) and of the sexuals/asexuals complex (lower half of each figure; sexuals on the left, asexuals on the right) in the case all parameters for $B$ and $U$ equal for Bobyrev and Schley model on panel $\mathrm{A}$ and $\mathrm{B}$, respectively. Horizontal and vertical axis corresponds to the space and time, respectively. 
A)

B)
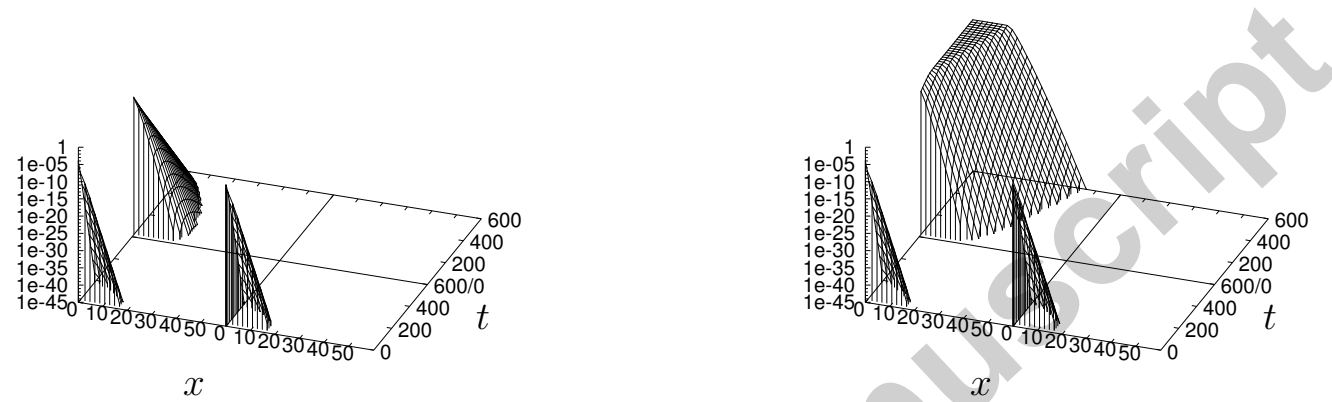

C)

D)

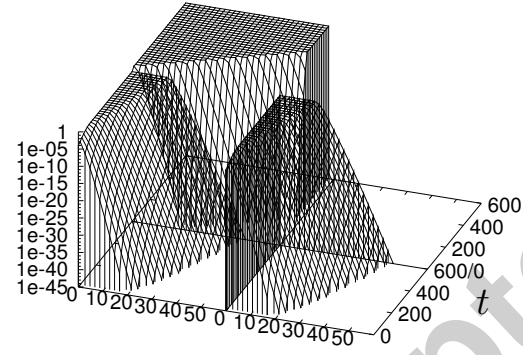

$x$

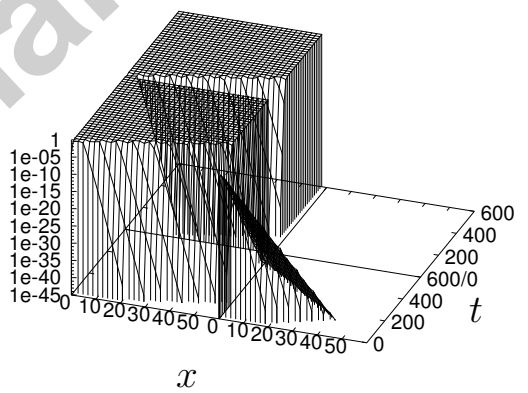

Figure 2: Spatial occupation of sexuals (upper half) and of the sexuals/asexuals complex (lower half of each figure; sexuals on the left, asexuals on the right) in Bobyrev model for different $\alpha_{B} / \alpha_{U}$ ratio $(0.6,1.0,2.0$ and 3.3) with all other parameters for $B$ and $U$ equal (more precisely, $\left.r=1, K=3, \alpha_{U}=1.5, \mu_{B}=\mu_{U}=1, \gamma_{B}=\gamma_{U}=1\right)$. 
A)

B)
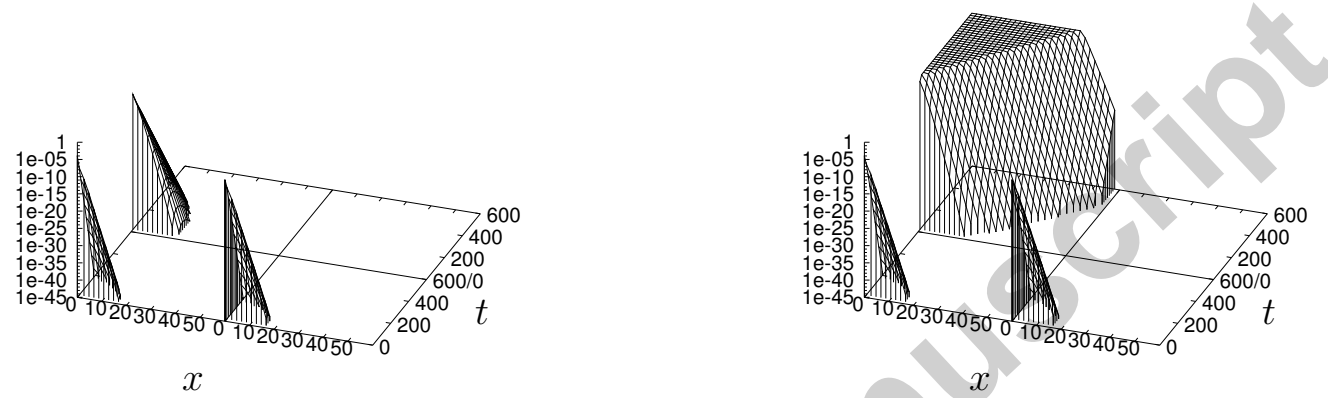

C)

D)
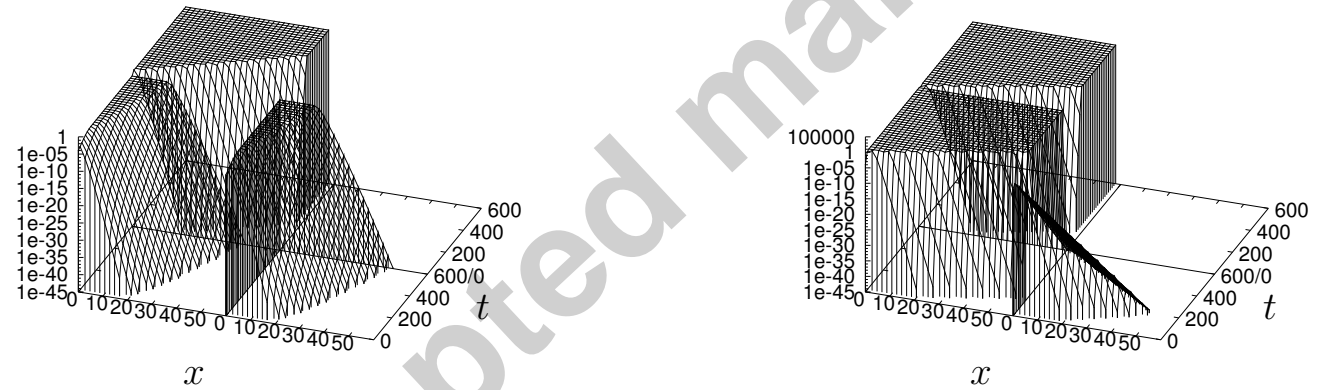

Figure 3: Spatial occupation of sexuals (upper half) and of the sexuals/asexuals complex (lower half of each figure; sexuals on the left, asexuals on the right) in Bobyrev model with different $\gamma_{B} / \gamma_{U}(0.5,1.3,2.0$ and 3.0) with and all other parameters for $B$ and $U$ equal (more precisely, $\left.r=1, K=3, \alpha_{B}=\alpha_{U}=1.5, \mu_{B}=\mu_{U}=1, \gamma_{U}=1\right)$. 
A)

B)
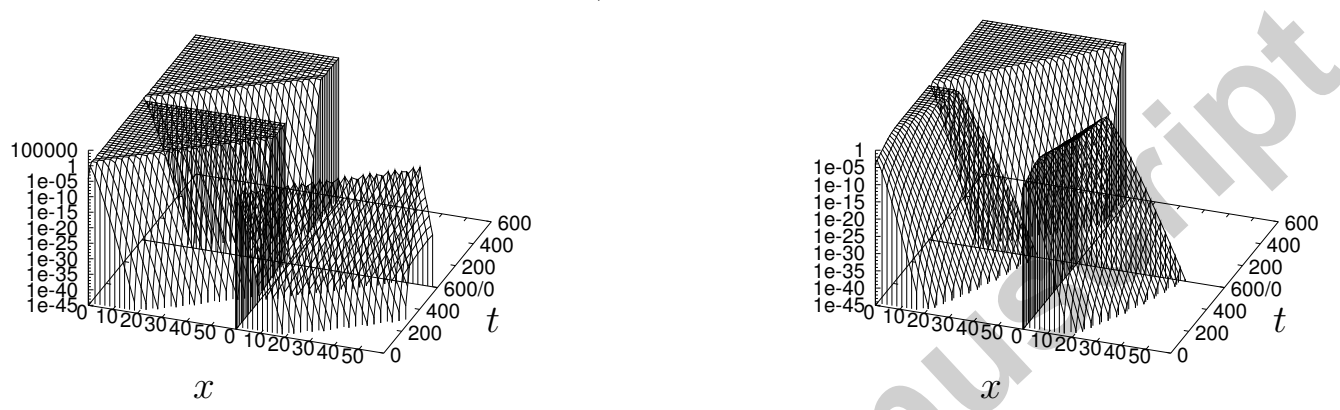

C)

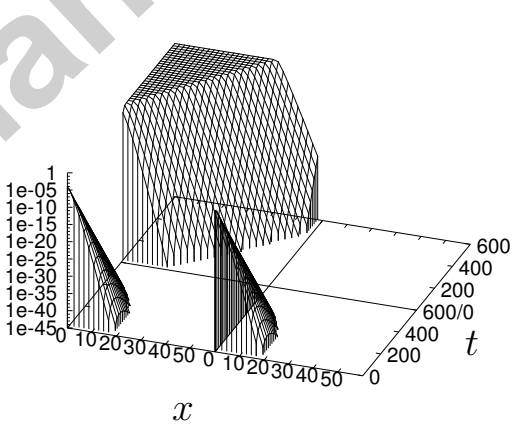

D)

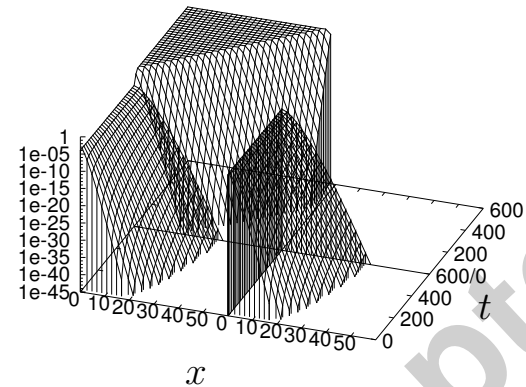

Figure 4: Spatial occupation of sexuals (upper half) and of the sexuals/asexuals complex (lower half of each figure; sexuals on the left, asexuals on the right) in Bobyrev model for different $\mu_{B} / \mu_{U}$ ratio $(0.05,0.45,0.5$ and 0.7$)$ with all other parameters for $B$ and $U$ equal (more precisely, $\left.r=1, K=3, \alpha_{B}=\alpha_{U}=1.5, \mu_{U}=1, \gamma_{B}=\gamma_{U}=1\right)$. 
A)

B)
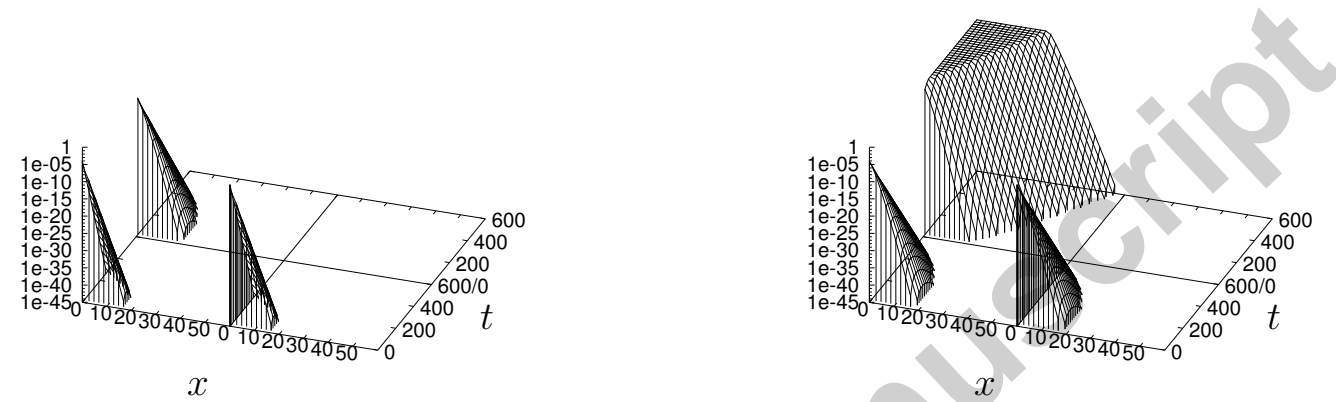

C)

D)
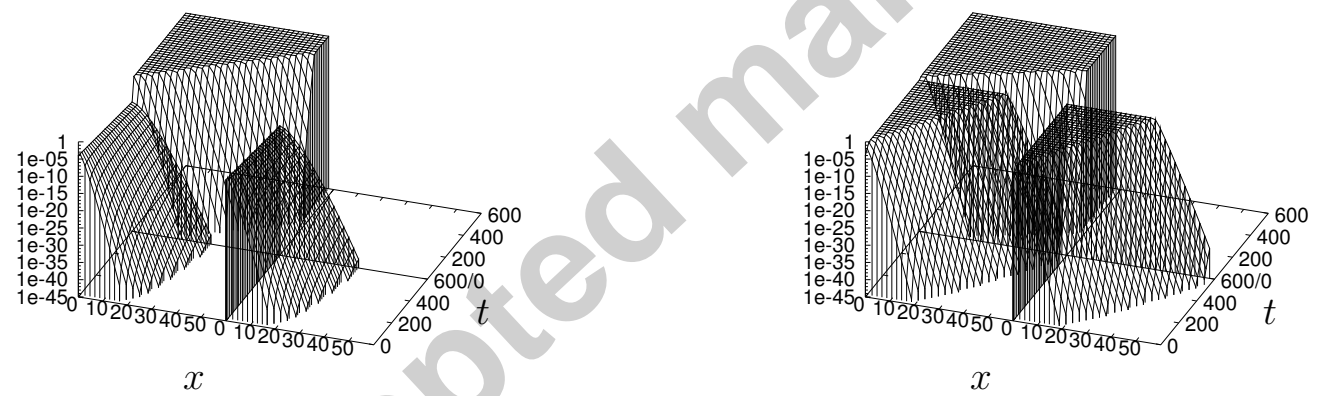

Figure 5: Spatial occupation of sexuals (upper half) and of the sexuals/asexuals complex (lower half of each figure; sexuals on the left, asexuals on the right) in Bobyrev model with different $K\left(0.8,1.7,2.6\right.$ and 3.6) with $\gamma_{B} / \gamma_{U}=2$, and all other parameters for $B$ and $U$ equal (more precisely, $\left.r=1, \alpha_{B}=\alpha_{U}=1.5, \mu_{B}=\mu_{U}=1, \gamma_{B}=2, \gamma_{U}=1\right)$. 
A)

B)
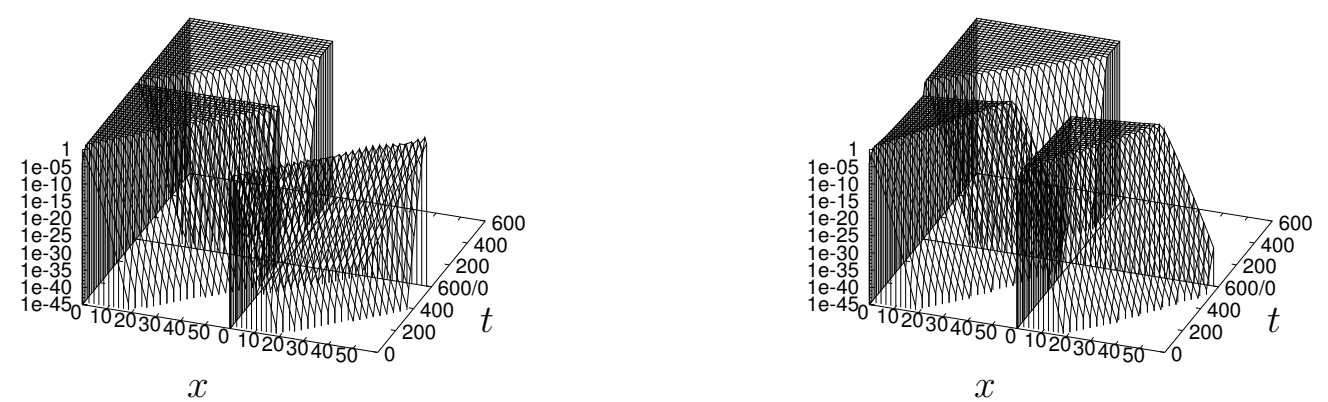

C)

$x$
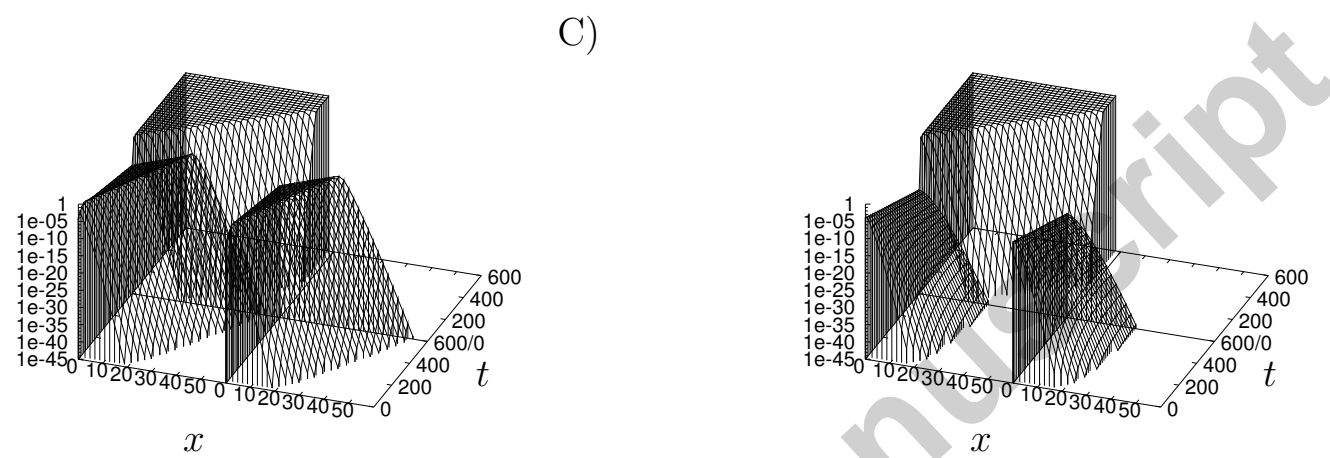

D)

Figure 6: Spatial occupation of sexuals (upper half) and of the sexuals/asexuals complex (lower half of each figure; sexuals on the left, asexuals on the right) in Schley model with different $\kappa$ $(0.5,1.15,2.0$ and 50) and all other parameters for $B$ and $U$ equal (more precisely, $\mu=0.1$, $\left.\beta=1, \eta_{B}=\eta_{U}=1\right)$.
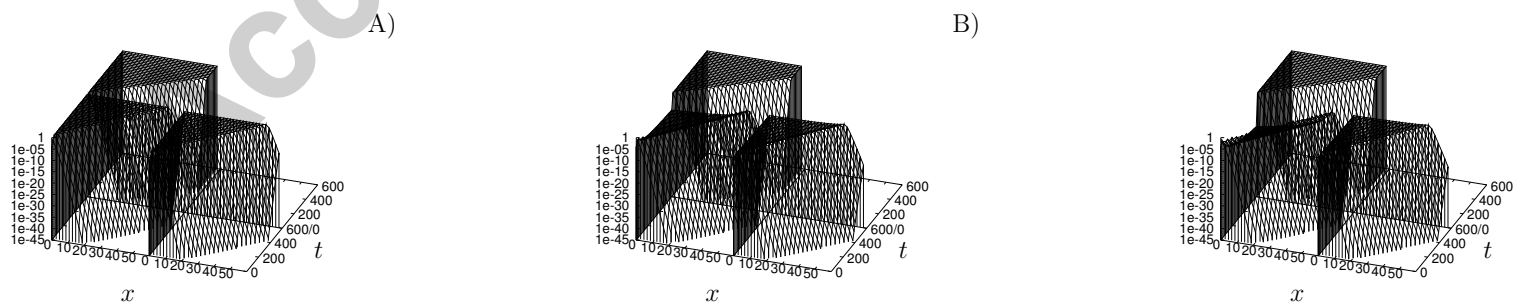

Figure 7: Spatial occupation of sexuals (upper half) and of the sexuals/asexuals complex (lower half of each figure; sexuals on the left, asexuals on the right) in Schley model with different $\eta_{U}$ (0.1, 3.0 and 10.0) and all other parameters for $B$ and $U$ equal (more precisely, $\mu=0.1, \kappa=1$, $\left.\beta=1, \eta_{B}=1\right)$. 
A)

B)
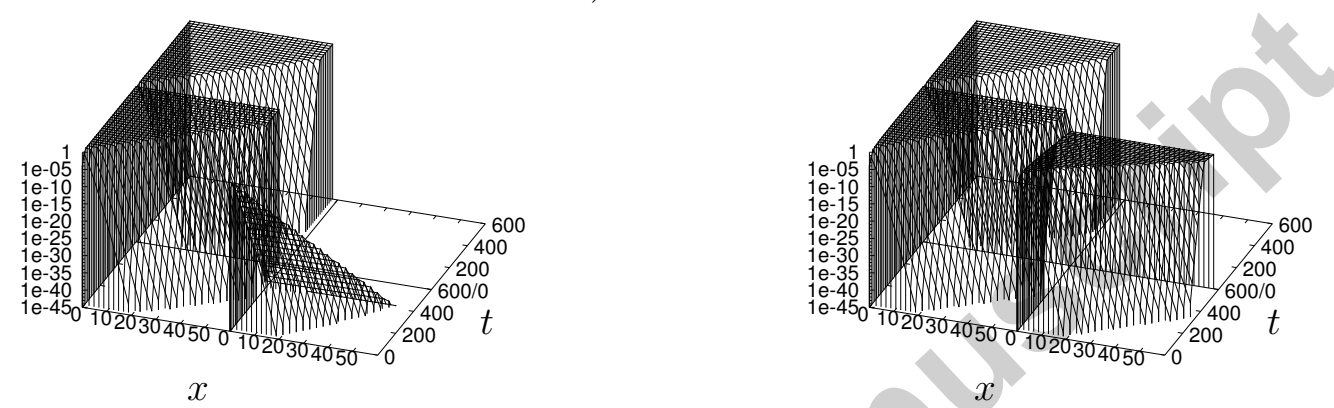

C)

D)
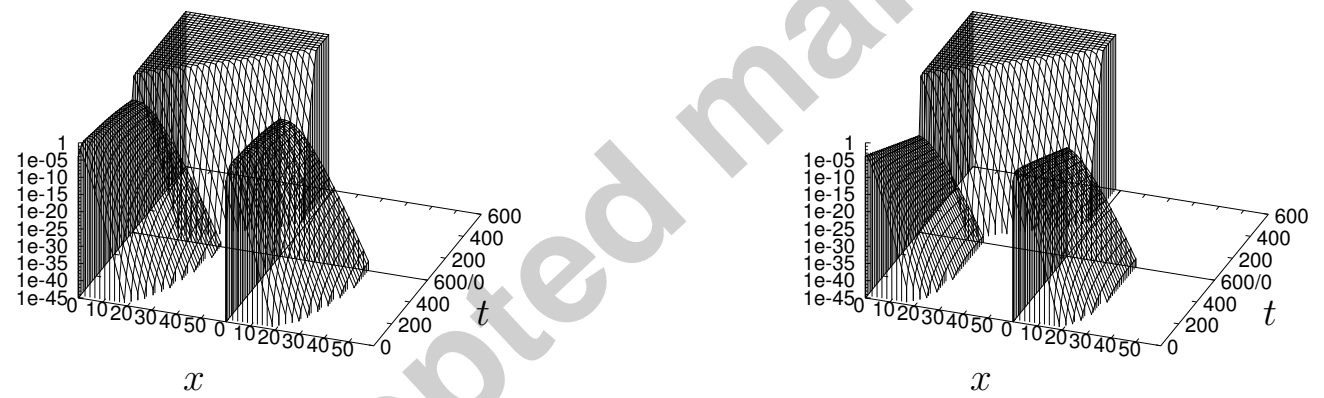

Figure 8: Spatial occupation of sexuals (upper half) and of the sexuals/asexuals complex (lower half of each figure; sexuals on the left, asexuals on the right) in Schley model with different $\beta$ $(0.5,0.95,1.1$ and 10.0) and all other parameters for $B$ and $U$ equal (more precisely, $\mu=0.1$, $\left.\kappa=1, \eta_{B}=\eta_{U}=1\right)$. 